\title{
DESARROLLO GERENCIAL
}

ÓRGANO DE DIFUSIÓN CIENTÍFICA DE LA UNIVERSIDAD SIMÓN BOLÍVAR

Colombia, Suramérica, diciembre 2013

http://portal.unisimonbolivar.edu.co:82/rdigital/desarrollogerencial/

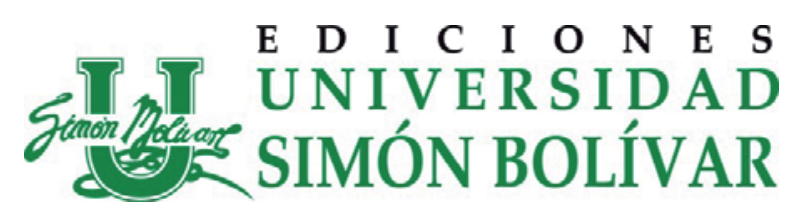

El editor y los autores son responsables de los artículos aquí publicados. Se autoriza la reproducción total o parcial de los artículos citando la fuente y el autor o los autores.

www.unisimon.edu.co

DESARROLLO GERENCIA4 Colombia | Vol. 5 No.2 lpp. 1-298| Dic. 2013| ISSN: 2145-147 


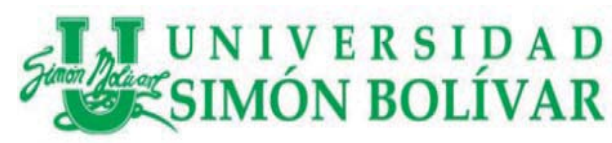

Autorizada por resolución No. 2624

31 de marzo de 1977

Del Ministerio de Educación Nacional

\title{
DIRECTIVO SALA GENERAL
}

Ana Bolívar de Consuegra, Presidenta

José Consuegra Higgins

Álvaro Castro Socarras

Eugenio Bolívar Romero

José Ignacio Consuegra Manzano

Rodolfo Pérez Vásquez, Secretario

RECTOR FUNDADOR

José Consuegra Higgins

RECTOR EJECUTIVO

José Consuegra Bolívar

\section{VICERRECTORÍA ACADÉMICA}

Sonia Falla

Barrantes

\section{VICERRECTORÍA ADMINISTRATIVA}
Eugenio Bolívar
Romero

\section{VICERRECTORÍA DE BIENESTAR}

Ana Bolívar de Consuegra

\section{VICERRECTORÍA DE PLANEACIÓN}
Ignacio Consuegra
Bolívar

\section{VICERRECTORÍA FINANCIERA}

Anita Consuegra
de Bayuelo

\author{
INSTITUTO DE INVESTIGACIONES \\ Directora \\ María Pérez Hernández \\ Subdirector \\ Ronald Álvarez Martínez \\ Jefe de Departamento de Publicaciones \\ Francisco Vásquez De la Hoz \\ Comité de Publicaciones \\ Comité de Editores
}




\section{DESARROLLO GERENCIAL}

ORGANO DE DIFUSIONN CIENTIFICA DE LA UNIVERSIDAD SIMONN BOLIVAR

Colombia, Suramérica, Junio 2012

http://portal.unisimonbolivar.edu.co:82/rdigital/desarrollogerencial/

\section{COMITÉ EDITORIAL COMITÉ CIENTÍFICO}

Ph.D. Manuel Garzón Dra. Rosa María Romero

Fundación para la Investigación y el Desarrollo

Educativo y Empresarial - Colombia

Ph.D. Ángel Vázquez Wilhelm

Universidad de Azcatpoalco - México

Ph.D. Victor Uribe Uran

University of Pittsburgh - E. U.

Dra. Irma Carrillo Flórez

Universidad Autónoma de Aguascalientes - México

Ph.D. Edwin Tarapuez

Universidad del Quindío - Colombia

Ph.D. León Cabello Cervantes

Universidad Autónoma de Querétaro - México

Mg. Christian Figueroa

Universidad Nacional de Costa Rica - Costa Rica
Universidad Autónoma de Querétaro - México

Dra. Lourdes Osorio Bayter

Universidad del Rosario - Colombia

Dra. Alejandra Urbiola Solís

Universidad Autónoma de Querétaro - México

Dr. Amado Olivares Leal

Universidad Sonora, México

Ph.D. Julio Cesar Acosta Prado

Universidad Externado de Colombia

Mg. Ronal Cancino

Universidad de la Frontera - Chile

Mg. Carlos Mario Durango

Universidad Pontificia Bolivariana de Medellín -

Colombia

Mg. Fernando Jaramillo Betacourt

Universidad Nacional de Antioquia - Colombia 


\section{DESARROLLO GERENCIAL}

ORGANO DE DIFUSIÓN CIENTIFICA DE LA UNIVERSIDAD SIMÓN BOLIVAR

Colombia, Suramérica, Junio 2012

http://portal.unisimonbolivar.edu.co:82/rdigital/desarrollogerencial/

\section{EQUIPO EDITORIAL}

\section{Directora}

Mg. Adriana Uribe Uran

Directora Programa de

Administración De Empresas

Editora

Mg. Pabla Peralta Miranda

Miembro del grupo de investigación

Gestión Organizacional

Coeditora

Mg. Viviana Cervantes Atia

Docente

Sede Barranquilla

Coeditora

Mg. Mariluz Ordoñez Santos

Docente, sede Cúcuta
Administración OJS y Soporte Tecnológico

Mg. Adriana Iglesias Solano

Correctores de Estilos y

Traducciones

Antonio Silvera Arenas

Elvira Días Barreto

Andrew White

Coeditora

Mg. Diana Escamilla Narváez

Directora Programa Administración

Administración de Empresas, sede

Cúcuta

Asistente del Editor

Alexsandra Cabarcas Ramos

\section{Editores}

Viviana Cervantes Atia

Pabla Peralta Miranda

\section{Asesor Experto Del Equipo \\ Editorial \\ Ernesto Ravelo Contreras}

